\section{The relation between paracetamol use and asthma: a GA²LEN European case-control study}

S. Shaheen, J. Potts, L. Gnatiuc, J. Makowska, M.L. Kowalski, G. Joos, T. van Zele, Y. van Durme, I. De Rudder, S. Wöhrl, J. Godnic-Cvar, L. Skadhauge, G. Thomsen,

T. Zuberbier, K.C. Bergmann, L. Heinzerling, M. Gjomarkaj, A. Bruno, E. Pace,

S. Bonini, W. Fokkens, E.J.M. Weersink, C. Loureiro, A. Todo-Bom, C.M. Villanueva,

C. Sanjuas, J-P. Zock, C. Janson and P. Burney

ABSTRACT: Studies from the UK and USA suggest that frequent use of paracetamol (acetaminophen) may increase the risk of asthma, but data across Europe are lacking.

As part of a multicentric case-control study organised by the Global Allergy and Asthma European Network (GA ${ }^{2}$ LEN), it was examined whether or not frequent paracetamol use is associated with adult asthma across Europe. The network compared 521 cases with a diagnosis of asthma and reporting of asthma symptoms within the last 12 months with 507 controls with no diagnosis of asthma and no asthmatic symptoms within the last 12 months across 12 European centres. All cases and controls were selected from the same population, defined by age (20$45 \mathrm{yrs}$ ) and place of residence.

In a random effects meta-analysis, weekly use of paracetamol, compared with less frequent use, was strongly positively associated with asthma after controlling for confounders. There was no evidence for heterogeneity across centres. No association was seen between use of other analgesics and asthma.

These data add to the increasing and consistent epidemiological evidence implicating frequent paracetamol use in asthma in diverse populations.

\section{KEYWORDS: Asthma, Global Allergy and Asthma European Network, paracetamol}

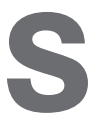
ince the year 2000, evidence has been accumulating that frequent paracetamol (acetaminophen) use is associated with asthma in adults. Following the initial finding in a population-based case-control study of adult asthma in London (UK) [1], other studies have replicated these observations. The Nurses Health Study, in the USA, reported a positive prospective association between paracetamol use and incident asthma in females [2]. Positive crosssectional associations have also been found in Ethiopia in relation to allergic symptoms [3], and in the US National Health and Nutrition Examination Survey in relation to asthma and COPD [4]. Positive geographical correlations between paracetamol sales and asthma, wheeze, rhinitis and bronchial hyperresponsiveness have also been observed in adults across Europe [5], but, given the limitations of ecological studies, the aim of the present study was to determine whether or not an association between frequent paracetamol use and adult asthma could be confirmed in individuals living in different environments. As part of a multicentric casecontrol study organised by the Global Allergy and Asthma European Network (GA $\left.{ }^{2} L E N\right)$, which was set up primarily to assess the relation of plasma selenium to adult asthma, the relation between analgesic use and asthma was examined in European adults.

\section{METHODS}

The present study was a case-control study. The cases were aged 20-45 yrs, lived in a defined area and had both a self-reported diagnosis of asthma and a history of either wheezing, shortness of breath or waking at night with breathlessness within the previous 12 months [6]. The controls lived in the same area, were aged 20-45 yrs and had neither a diagnosis of asthma nor any of the three symptoms. In the first instance, cases and controls were identified from a population-based survey, mostly through a simple screening questionnaire sent by post. However, these surveys
AFFILIATIONS

Author affiliation details can be found in the Acknowledgements section

Participating centres are also listed in the Acknowledgements.

CORRESPONDENCE

S. Shaheen

National Heart and Lung Institute

Imperial College London

Emmanuel Kaye Building

Manresa Road

London SW3 6LR

UK

Fax: 442073518322

E-mail: s.shaheen@imperial.ac.uk

Received:

March 132008

Accepted after revision:

June 132008

\section{SUPPORT STATEMENT}

The coordination of this study was supported by the European Commission (Brussels, Belgium; grant number FOOD-CT-2004506378).

STATEMENT OF INTEREST Statements of interest for S. Wöhrl, S. Bonini and P. Burney and the study itself can be found at www.erj.ersjournals.com/misc/ statements.shtml

European Respiratory Journal Print ISSN 0903-1936 Online ISSN 1399-3003 
were not always large enough to locate adequate numbers of cases, and further cases could be recruited from clinics providing that they met the above criteria and were not being treated in the clinic for another atopic condition. Each centre was asked to recruit 50 cases and 50 controls.

Once identified, the participants were invited to complete a longer administered questionnaire and had their height and weight measured. Skin tests were undertaken using ALK (Madrid, Spain) and ALK-Abelló (Hørsholm, Denmark) reagents directed against timothy grass, cat dander, Dermatophagoides farinae, olive, birch, Parietaria, Alternaria and histamine $\left(10 \mathrm{mg} \cdot \mathrm{mL}^{-1}\right)$ and diluent controls. Atopy was defined by the very sensitive criterion of any atopic weal of greater diameter than that of the diluent control in the presence of a positive histamine control. Asthma cases who showed night-time symptoms twice or less per month, trouble breathing less than once a day, a forced expiratory volume in one second of $\geqslant 80 \%$ of the predicted value and were using at most a medium dose of inhaled steroid were classified as having intermittent and mild persistent disease; those with more severe symptoms, worse lung function and using higher doses of steroids were classified as having moderate or severe persistent disease.

Central training was given to the staff in each centre prior to involvement with the main data collection. Questionnaires were forward- and back-translated, and the original questionnaires and the back-translations compared and reconciled. Participants were asked how often they took paracetamol and how often they took other painkillers not containing paracetamol (such as aspirin or ibuprofen). The options were: $<1$ day $\cdot$ week $^{-1}$, 1-2 days $\cdot$ week $^{-1}$, 3-4 days $\cdot$ week $^{-1}$, 5-6 days. week $^{-1}$ and daily. However, since very frequent use turned out to be uncommon, the top four categories were collapsed down into one category of weekly for the main analyses. Socioeconomic status was assessed from the current or last occupation and classified as professional, managerial, skilled, semi-skilled, unskilled or student. Smoking status was classified as: never-smoker exposed to environmental tobacco smoke for $<1 \mathrm{~h} \cdot$ day $^{-1}$; never-smoker exposed to environmental tobacco smoke for $\geqslant 1 \mathrm{~h} \cdot$ day $^{-1}$; ex-smokers; and current smokers. Participants were asked whether or not they took regular vitamin or mineral supplements. After examining the distributions of the variables, logistic regression was used to estimate the risk of being a case in each centre. These analyses controlled for the potential confounders sex, age, smoking, socioeconomic status, supplement use and body mass index (in kilograms per metre squared). After initial within-centre analyses, the results were combined across centres using random effects metaanalysis.

Local ethical committee approval was obtained in each centre and approval was given by the Norwegian Data Inspectorate (Oslo, Norway). Each participant was provided with an information sheet explaining the study, and signed a consent form prior to taking part.

\section{RESULTS}

The 12 centres recruited a total of 521 cases and 507 controls. Clinic visits occurred between May 2005 and May 2007.
Table 1 shows the distribution of potential confounders in cases and controls. Cases were more likely than controls to be overweight or obese and female, and less likely to be working in professional jobs. Smoking history, supplement use and mean age were similar in the two groups. The prevalence of atopy in cases and controls was 82 and 36\%, respectively. Amongst cases, $54 \%$ were classified as having intermittent or mild persistent disease and the remainder as having moderate or severe persistent disease.

Table 2 shows the frequency of use of paracetamol and other analgesics by centre and case-control status. In Italy (Palermo and Rome), no participants reported weekly paracetamol use, and the highest frequency of weekly use occurred in Amsterdam (the Netherlands). Overall, 8.9\% of individuals reported taking paracetamol at least once a week. This was more common in cases $(13.2 \%)$ than in controls $(4.5 \%)$. In the main paracetamol analyses, three centres were dropped (Vienna (Austria), Rome and Palermo) as too few individuals were taking paracetamol weekly and this led to a failure of the regression model to converge. Similarly, Amsterdam and Barcelona (Spain) had to be omitted from the analyses of other analgesic use for the same reason.

Table 3 shows the unadjusted and adjusted odds ratios (ORs) for asthma according to paracetamol use in each centre, and in all centres combined. This shows that weekly paracetamol use (compared with less frequent use) was positively associated with asthma, and the overall effect estimate became stronger after controlling for confounders (adjusted OR 2.87; 95\%

\begin{tabular}{|c|c|c|c|}
\hline \multirow[t]{2}{*}{ TABLE 1} & \multicolumn{3}{|c|}{$\begin{array}{l}\text { Distribution of potential confounders in cases } \\
\text { and controls }\end{array}$} \\
\hline & Controls & Cases & Total \\
\hline Total & 507 (49.3) & $521(50.7)$ & 1028 \\
\hline \multicolumn{4}{|l|}{ Body mass index } \\
\hline$<20 \mathrm{~kg} \cdot \mathrm{m}^{-2}$ & $51(10.1)$ & $52(10.0)$ & $103(10.0)$ \\
\hline $20-25 \mathrm{~kg} \cdot \mathrm{m}^{-2}$ & $270(53.3)$ & $238(45.7)$ & $508(49.4)$ \\
\hline $25-30 \mathrm{~kg} \cdot \mathrm{m}^{-2}$ & $135(26.6)$ & $156(29.9)$ & $291(28.3)$ \\
\hline$\geqslant 30 \mathrm{~kg} \cdot \mathrm{m}^{-2}$ & $51(10.1)$ & $73(14.0)$ & $124(12.1)$ \\
\hline Male sex & $239(47.1)$ & $206(39.5)$ & 445 (43.3) \\
\hline Age yrs & $34.7 \pm 7.48$ & $32.8 \pm 7.37$ & $33.7 \pm 7.48$ \\
\hline \multicolumn{4}{|l|}{ Socioeconomic status } \\
\hline Professional & $242(47.7)$ & $186(35.7)$ & $428(41.6)$ \\
\hline Managerial & $29(5.7)$ & $28(5.4)$ & $57(5.5)$ \\
\hline Skilled & $139(27.4)$ & $186(35.7)$ & 325 (31.6) \\
\hline Semi-skilled & $29(5.7)$ & $29(5.6)$ & $58(5.6)$ \\
\hline Unskilled & $27(5.3)$ & $39(7.5)$ & $66(6.4)$ \\
\hline Students & $38(7.5)$ & $43(8.3)$ & $81(7.9)$ \\
\hline \multicolumn{4}{|l|}{ Smoking } \\
\hline Never-smoker & $139(27.4)$ & $142(27.3)$ & $281(27.3)$ \\
\hline $\begin{array}{l}\text { Exposed to environmental } \\
\text { tobacco smoke }\end{array}$ & $142(28.0)$ & $150(28.8)$ & $292(28.4)$ \\
\hline Ex-smoker & 93 (18.3) & $93(17.9)$ & $186(18.1)$ \\
\hline Current smoker & $133(26.2)$ & $136(26.1)$ & $269(26.2)$ \\
\hline Supplement use & $106(20.9)$ & $123(23.6)$ & 229 (22.3) \\
\hline
\end{tabular}


TABLE 2 Frequency of paracetamol and other analgesic use by centre and case-control status

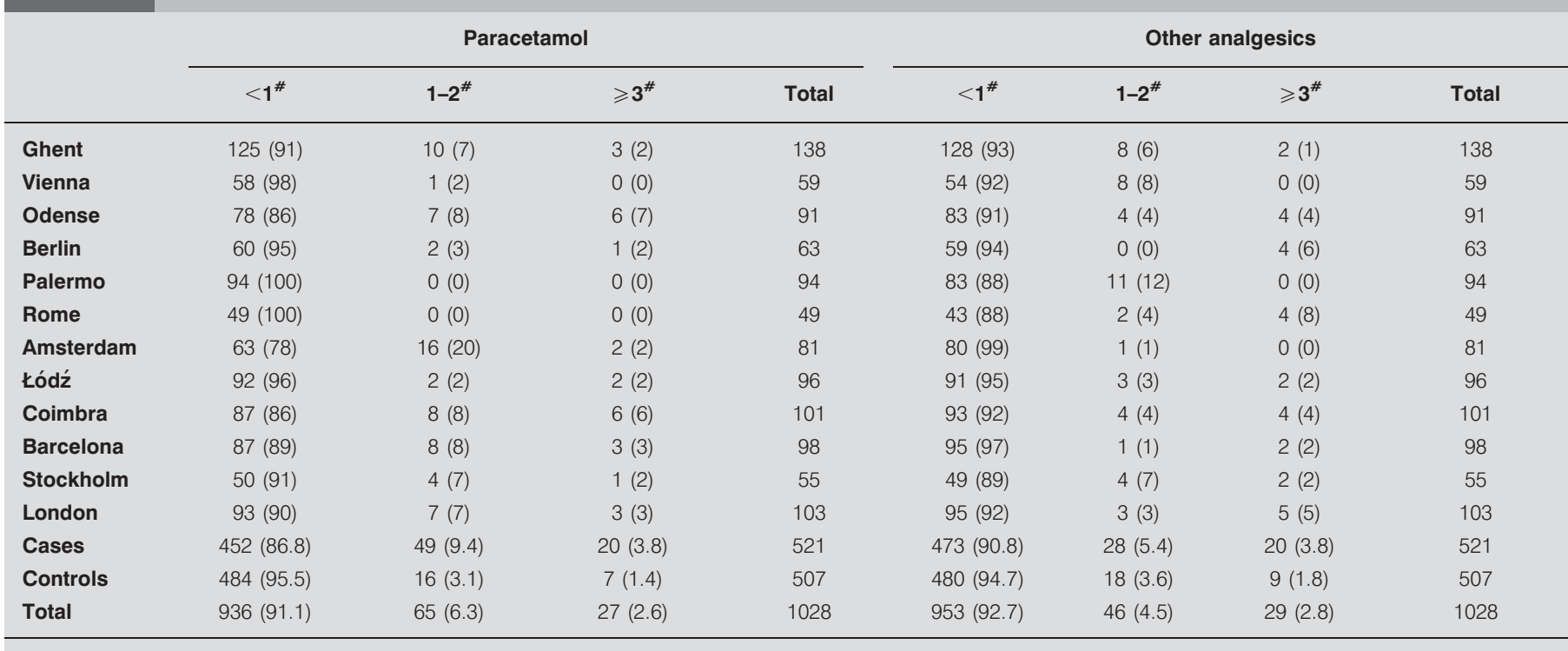

Data are presented as n (\%) or n. ${ }^{\#}$ : days $\cdot$ week $^{-1}$.

confidence interval (CI) 1.49-5.37; $\mathrm{p}=0.002)$. There was no significant heterogeneity between centres $(p=0.61)$. The metaanalysis of the adjusted effects is summarised in figure 1.

Table 4 shows the unadjusted and adjusted ORs for asthma according to other analgesic use in each centre, and in all centres combined. The overall adjusted effect estimate showed a nonsignificant positive association with asthma, and, again, there was no significant heterogeneity between centres. The meta-analysis of the adjusted effects is summarised in figure 2 . When paracetamol and other analgesic use were included together in the regression model, the overall adjusted effect estimate for paracetamol was only slightly attenuated (OR 2.66; $95 \%$ CI 1.33-5.33; $p=0.006)$. Of the cases, $75 \%$ reported rhinitis.
If rhinitis was included in the model, the adjusted OR was reduced a little to 2.39 (95\% CI 1.06-5.37; $\mathrm{p}=0.036)$.

\section{DISCUSSION}

In the present multicentric study across Europe, a positive association between frequent use of paracetamol and adult asthma has been confirmed, in keeping with previous studies of individuals from the UK [1] and USA [2, 4], and ecological observations across Europe [5]. Weekly users of paracetamol were more likely than those taking the drug less often to exhibit asthma, and there was no significant heterogeneity in this result between centres. No significant relation was seen between use of other analgesics and asthma.

TABLE 3 Association of asthma with frequency of paracetamol use

$$
<1 \text { day. } \text { week }^{-1} \text { OR }
$$

$\geqslant 1$ day $\cdot$ week $^{-1}$

\begin{tabular}{cccc}
\hline Unadjusted OR (95\% Cl) & p-value & Adjusted ${ }^{\#}$ OR (95\% Cl) & p-value \\
$4.61(0.98-21.66)$ & 0.05 & $5.20(0.97-27.77)$ & 0.05 \\
$1.93(0.55-6.79)$ & 0.31 & $2.88(0.53-15.58)$ & 0.22 \\
$3.00(0.26-34.95)$ & 0.38 & $1.86(0.07-47.03)$ & 0.71 \\
$1.38(0.48-3.94)$ & 0.55 & $1.17(0.32-4.26)$ & 0.82 \\
$2.63(0.26-26.26)$ & 0.41 & $4.30(0.16-117.32)$ & 0.39 \\
$14.59(1.83-116.39)$ & 0.01 & $16.11(1.25-207.41)$ & 0.03 \\
$12.89(1.58-105.18)$ & 0.02 & $14.34(1.25-164.53)$ & 0.03 \\
$1.88(0.19-18.23)$ & 0.59 & $2.77(0.11-68.54)$ & 0.53 \\
$1.67(0.44-6.31)$ & 0.45 & $1.73(0.36-8.34)$ & 0.49 \\
$2.57(1.51-4.37)$ & 0.001 & $2.87(1.49-5.37)$ & 0.002 \\
\hline
\end{tabular}

In testing for heterogeneity (Cochran's Q) of the combined results that contributed towards the overall mean result, Chi-squared=7.55 (8 degrees of freedom (df) $\mathrm{p}=0.48$ ) for unadjusted values and 6.29 ( $8 \mathrm{df} ; \mathrm{p}=0.61)$ for adjusted values. OR: odds ratio; Cl: confidence interval. ${ }^{\#}$ : adjusted for age, sex, body mass index, socioeconomic status, smoking and supplement use. 


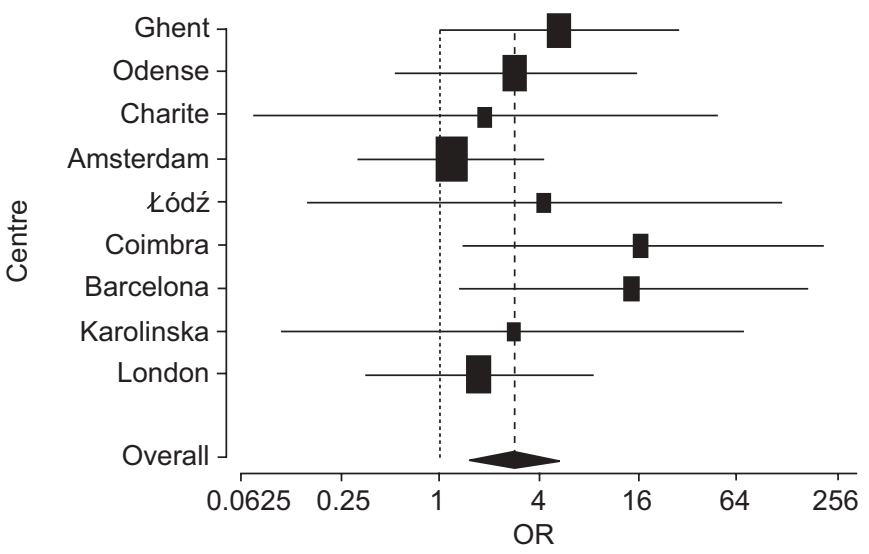

FIGURE 1. Forest plot showing meta-analysis of asthma risk according to weekly paracetamol use (versus less than weekly use). Data are presented as adjusted odds ratios (ORs; (size reflects weighting)), using a random effect model, and $95 \%$ confidence intervals (horizontal bars). The centre of the diamond indicates the combined mean effect of the studies (OR 2.87; -..---) and its extremities, the $95 \%$ confidence interval (……... line of no effect).

A strength of the present study is that the methods and definitions used were standardised across centres. However, it had some limitations. First, most individuals took paracetamol $<1$ day.week ${ }^{-1}$, and the questions on analgesic use permitted neither the categorisation of infrequent use in more detail nor distinction of those who never took paracetamol. Furthermore, there were insufficient numbers of weekly users to permit the further breakdown of this category. Hence it was not possible to determine whether or not a dose-response relation was present. Secondly, the cross-sectional nature of the association makes a causal interpretation difficult, although taking paracetamol for asthma symptoms (reverse causation) seems unlikely, and findings from a cohort study in the USA have indicated that frequent paracetamol use preceded the development of adult-onset asthma [2]. Thirdly,

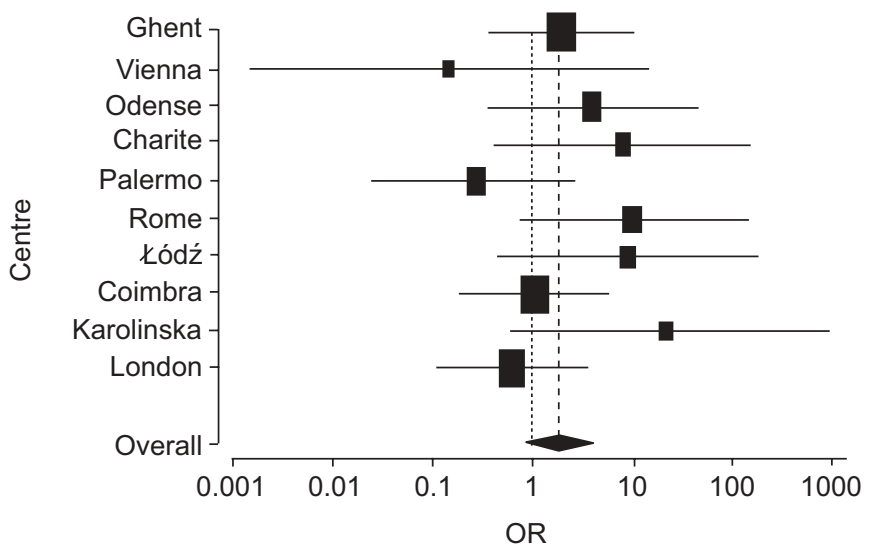

FIGURE 2. Forest plot showing meta-analysis of asthma risk according to weekly use of painkillers other than paracetamol (versus less than weekly use). Data are presented as adjusted odds ratios (ORs; $\mathbf{\square}$ (size reflects weighting)), using a random effect model, and 95\% confidence intervals (horizontal bars). The centre of the diamond indicates the combined mean effect of the studies (OR 1.85; -...--) and its extremities, the $95 \%$ confidence interval ( $. \cdots \cdots \cdots . . .$. line of no effect).

separate questions about frequency of use of aspirin and nonsteroidal anti-inflammatory drugs were not asked, although these are likely to have been the most commonly used non-paracetamol-containing analgesics. Were the explanation of the paracetamol findings avoidance of aspirin and nonsteroidal anti-inflammatory drugs by asthmatic individuals, a negative association between other analgesic use and asthma would have been expected, which was not the case.

Finally, in an observational study of this kind, the possibility that the association between paracetamol use and asthma has arisen through bias or unmeasured or residual confounding cannot be ruled out. However, cases and controls were selected from similar populations in each centre, making selection bias less likely. Information was not collected on indications for

\section{TABLE 4 Association of asthma with other painkiller use}

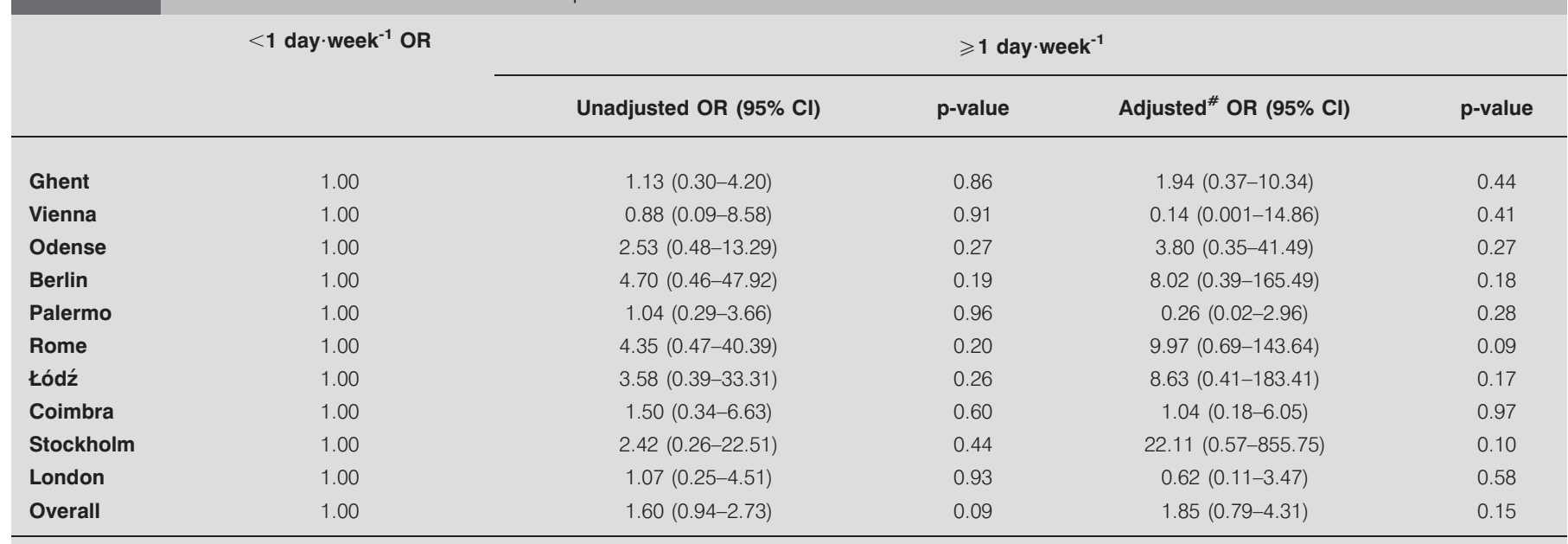

In testing for heterogeneity (Cochran's Q) of the combined results that contributed towards the overall mean result, Chi-squared=3.84 (9 degrees of freedom (df); $\mathrm{p}=0.92)$ for unadjusted values and 11.13 (9 df; $\mathrm{p}=0.27$ ) for adjusted values. OR: odds ratio; $\mathrm{Cl}$ : confidence interval. ${ }^{*}$ : adjusted for age, sex, body mass index, socioeconomic status, smoking and supplement use. 
use, however, and, although it is possible that a minority of cases were taking paracetamol for conditions that are more common in people with asthma, such as rhinitis or migraine, it seems unlikely that such confounding would be of sufficient magnitude to account for the effect estimate for frequent paracetamol use. A previous case-control study indicated that the majority of adults with asthma take weekly paracetamol for non-migrainous headache and musculoskeletal complaints, and only a minority take it for migraine or rhinitis, at least in the UK [1]. Furthermore, when rhinitis was controlled for in the present study, the overall effect estimate was only attenuated a little, indicating minor confounding, but suggesting that use of paracetamol for rhinitis is unlikely to be the major explanation for the association between paracetamol use and asthma.

It has previously been suggested that glutathione depletion in the airways and increased oxidative stress may be the mechanism underlying the link between frequent paracetamol use and asthma [1]. Although evidence is lacking regarding the effects of therapeutic doses of paracetamol on airway glutathione levels, in vitro studies have shown that clinically relevant doses of paracetamol can deplete intracellular reduced glutathione levels in animal alveolar macrophages and type 2 pneumocytes [7] and human pulmonary macrophages in vitro [8], and that depletion of glutathione in antigen-presenting cells leads to preferential type-2 T-helper cell responses [9]. Furthermore, an in vivo study in humans demonstrated reductions in the antioxidant capacity of the blood following maximum therapeutic doses of paracetamol for 2 weeks [10], although reduced glutathione levels were not measured. The present authors, therefore, believe that frequent therapeutic doses of paracetamol may, in susceptible individuals, have adverse effects on the lung, through either oxidative damage to the airways or promotion of atopic responses, with consequences for asthma.

Given the increasing and consistent epidemiological evidence implicating paracetamol in adult asthma across diverse populations, and the high prevalence of asthma and paracetamol use in some countries, there is now a need to carry out suitable intervention studies in order to determine whether or not the link is causal.

\section{ACKNOWLEDGEMENTS}

The authors would like to thank ALK (Madrid, Spain) and ALK-Abelló (Hørsholm, Denmark) for donating the Soluprick study allergens.

Author affiliation details are as follows. S. Shaheen, J. Potts, L. Gnatiuc and P. Burney: National Heart and Lung Institute, Imperial College, London, UK. J. Makowska and M.L. Kowalski: Medical University, Łódź, Poland. G. Joos, T. van Zele, Y. van Durme and I. De Rudder: Ghent University, Ghent, Belgium. S. Wöhrl and J. Godnic-Cvar: Medical University of Vienna, Vienna, Austria. L. Skadhauge and G. Thomsen: Odense University Hospital, Odense, Denmark. T. Zuberbier, K.C. Bergmann and L. Heinzerling: AllergieCentrum-Charité, Charité - Universitätsmedizin Berlin, Germany. M. Gjomarkaj, A. Bruno and E. Pace: National Research Council, Palermo, Italy. S. Bonini: National Research Council, Rome, Italy. W. Fokkens and E.J.M. Weersink: Academic Medical Centre, Amsterdam, the Netherlands. C. Loureiro and A. Todo-Bom: University of Coimbra,
Coimbra, Portugal. C.M. Villanueva and J-P. Zock: Municipal Institute of Medical Research, Barcelona, Spain; Centre for Research in Environmental Epidemiology, Barcelona, Spain; and CIBER Epidemiología y Salud Pública, Barcelona, Spain. C. Sanjuas: Municipal Institute of Medical Research, Barcelona, Spain; CIBER Epidemiología y Salud Pública, Barcelona, Spain; and Servei de Pneumologia, Hospital del Mar, Barcelona, Spain. C. Janson: Uppsala University, Uppsala, Sweden.

Principal participants: the present article was drafted by S. Shaheen and J. Potts (National Heart and Lung Institute, Imperial College, London, UK) on behalf of the Selenium and Asthma Research Integration project.

Co-ordinating centre (National Heart and Lung Institute, Imperial College, London, UK): P. Burney, J. Phillips, J. Potts, L. Gnatiuc, and S. Shaheen.

Participating centres were as follow. Ghent University (Ghent, Belgium): G. Joos, P. Van Cauwenberge, T. van Zele, K. Verbruggen, Y. van Durme, and I De Rudder; Medical University of Vienna (Vienna, Austria): S. Wöhrl, J. GodnicCvar, and B. Salameh; Odense University Hospital (Odense, Denmark): L. Skadhauge, and G. Thomsen; Allergie-CentrumCharité (Charité - Universitätsmedizin Berlin, Germany): T. Zuberbier, K.C. Bergmann, and L. Heinzerling; National Research Council (Palermo, Italy): M. Gjomarkaj, A. Bruno, and E. Pace; National Research Council (Rome, Italy): S. Bonini, M. Bresciani, and C. Gramiccioni; Academic Medical Centre (Amsterdam, the Netherlands): W. Fokkens, and E.J.M. Weersink; Medical University (Eódź, Poland): J. Makowska, and M.L. Kowalski; University of Coimbra (Coimbra, Portugal): C. Loureiro, and A. Todo-Bom; Barcelona (Spain): C.M Villanueva (Municipal Institute of Medical Research, Centre for Research in Environmental Epidemiology and CIBER Epidemiología y Salud Pública), C. Sanjuas (Municipal Institute of Medical Research, CIBER Epidemiología y Salud Pública and Servei de Pneumologia, Hospital del Mar), and J-P. Zock (Municipal Institute of Medical Research, Centre for Research in Environmental Epidemiology and CIBER Epidemiología y Salud Pública); Karolinska Institute (Stockholm, Sweden): B. Lundback, and C. Janson.

\section{REFERENCES}

1 Shaheen SO, Sterne JA, Songhurst CE, Burney PG. Frequent paracetamol use and asthma in adults. Thorax 2000; 55: 266-270.

2 Barr RG, Wentowski CC, Curhan GC, et al. Prospective study of acetaminophen use and newly diagnosed asthma among women. Am J Respir Crit Care Med 2004; 169: 836-841.

3 Davey G, Berhane Y, Duncan P, Golnar A-A, Britton J, Venn AJ. Use of acetaminophen and the risk of self-reported allergic symptoms and skin sensitization in Butajira, Ethiopia. J Allergy Clin Immunol 2005; 116: 863-868.

4 McKeever TM, Lewis SA, Smit HA, Burney P, Britton JR, Cassano PA. The association of acetaminophen, aspirin, and ibuprofen with respiratory disease and lung function. Am J Respir Crit Care Med 2005; 171: 966-971.

5 Newson RB, Shaheen SO, Chinn S, Burney PGJ. Paracetamol sales and atopic disease in children and adults: an ecological analysis. Eur Respir J 2000; 16: 817-823. 
6 Burney PG, Laitinen LA, Perdrizet S, et al. Validity and repeatability of the IUATLD (1984) Bronchial Symptoms Questionnaire: an international comparison. Eur Respir J 1989; 2: 940-945.

7 Dimova S, Hoet PH, Nemery B. Paracetamol (acetaminophen) cytotoxicity in rat type II pneumocytes and alveolar macrophages in vitro. Biochem Pharmacol 2000; 59: 1467-1475.

8 Dimova S, Hoet PH, Dinsdale D, Nemery B. Acetaminophen decreases intracellular glutathione levels and modulates cytokine production in human alveolar macrophages and type II pneumocytes in vitro. Int $J$ Biochem Cell Biol 2005; 37: 1727-1737.

9 Peterson JD, Herzenberg LA, Vasquez K, Waltenbaugh C. Glutathione levels in antigen-presenting cells modulate Th1 versus Th2 response patterns. Proc Natl Acad Sci USA 1998; 95: 3071-3076.

10 Nuttall SL, Khan JN, Thorpe GH, Langford N, Kendall MJ. The impact of therapeutic doses of paracetamol on serum total antioxidant capacity. J Clin Pharm Therap 2003; 28: 289-294. 